\title{
Clostridium Septicum Aortitis: A Fatal Diagnosis
}

\author{
Troy Wiedenbeck ${ }^{\mathrm{a}}$, Ramil Goel ${ }^{\mathrm{a}}$, Kathryn Bollina, Daniel Roberts ${ }^{\mathrm{a}}$, Jorge Alegria ${ }^{\mathrm{a}}$, \\ Krishnaswamy Chandrasekaran ${ }^{\text {a }}$, Fadi Shamoun ${ }^{\mathrm{a}, \mathrm{b}}$
}

\begin{abstract}
Infectious aortitis is a rare but deadly condition. Few cases are reported in the literature with clostriduim bacteria, with no consensus on treatment that could change the prognosis. Immediate evaluation and treatment with antibiotics is crucial, and surgery might be needed. GI malignancy could be the source of the pathogen. This case highlights the natural course of clostridium aortitis.
\end{abstract}

Keywords: Aortitis; Clostridium septicum; Infectious aortitis

\section{Introduction}

Primary infection of the aorta or aortic aneurysms has an overall incidence between $0.7 \%$ and $2.6 \%$; with Salmonella spp. representing the most commonly identified pathogens. A rare cause of aortic inflammation is $C$. septicum, which typically occurs in patients with multiple comorbidities [1]. C. septicum infection progresses very rapidly, with a mortality rate of approximately $79 \%$ in adults, typically occurring within 48 hours of infection [2].

\section{Case Report}

An 84-year-old male with a history of coronary artery disease, hypertension and type 2 diabetes mellitus was hospitalized for one week of progressive right lower quadrant abdominal pain, diarrhea, fever and chills. His evaluation was

\footnotetext{
Manuscript accepted for publication July 18, 2013

${ }^{a}$ Divisions of Cardiology and Hospital Internal Medicine, Mayo Clinic in Arizona, USA

${ }^{\mathrm{b}}$ Corresponding author: Fadi Shamoun, Divisions of Cardiology and Hospital Internal Medicine, Mayo Clinic in Arizona, USA.

Email: shamoun.fadi@mayo.edu

doi: http://dx.doi.org/10.4021/jmc1402w
}

suggestive of enterocolitis with computed tomography of the abdomen revealing a circumferential thickening of the distal ileum and multiple enlarged ileocolic lymph nodes (Fig. 1). At that time he refused a colonoscopy or further evaluation and was treated empirically with oral ciprofloxacin and metronidazole and dismissed in stable condition.

Five days after completing his antibiotic regimen he presented to the emergency department with recurrent abdominal pain, fever, chills and hypotension. A computed tomography of the abdomen and pelvis revealed new mural gas extending through the descending aorta to the infrarenal aorta with associated periaortic fat stranding. Blood cultures grew Clostridium septicum. The patient declined surgical intervention. He was initially treated with empiric intravenous antimicrobials including vancomycin, voriconazole, levofloxacin, and pipercillin-tazobactam, but ultimately chose palliation and was discharged to hospice care with oral metronidazole. The patient passed away shortly thereafter.

\section{Discussion}

Infectious aortitis due to $C$. septicum is a very rare and often lethal [3]. C. septicum is a Gram-positive, motile bacillus whose ability to metastasize and invade tissues is facilitated by its flagella and the secretion of alpha-toxin, a hemoly$\sin [1]$. The classic CT finding of intramural air in the aorta made the diagnosis and the infectious organism was confirmed by blood culture in our patient. A recent review of the literature identified 26 such cases, of which $58 \%$ had isolated abdominal aortic segment involvement. Early treatment with intravenous antibiotics followed by definitive surgical therapy is required for optimal outcomes. The overall mortality rate is $64 \%$, with a 6 -month mortality of $100 \%$ in those who do not undergo surgical intervention [4]. Early radiographic indicators of aortitis include soft tissue prominence with increased contrast enhancement surrounding the involved segment. Intramural and peri-aortic emphysema is indicative of more advanced disease. Serial imaging of the aorta might be needed to monitor for aneurysmal transformation. The natural history of infectious aortitis is that of rapid aneurismal transformation, occurring in as little as 1 to 3 weeks, 


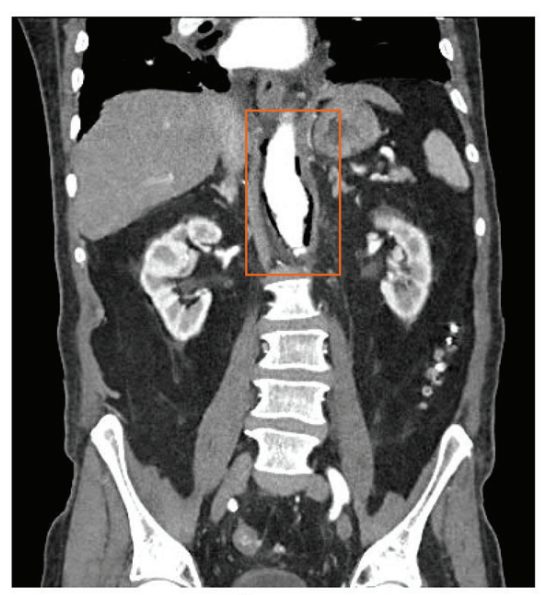

a

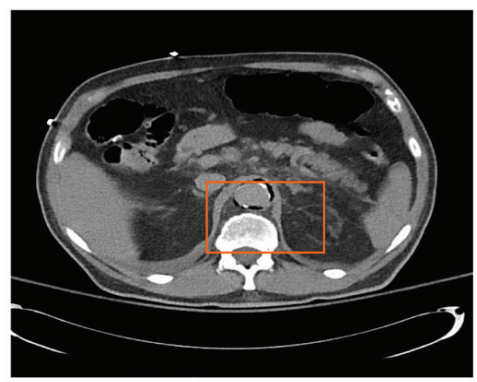

b

Figure 1. a). Cross-sectional and coronal views of the aorta demonstrating intramural gas seen as a dark region between the aortic wall and the contrast-filled lumen, b). and between the aortic wall and the intimal calcium in the non-contrast image.

with subsequent rupture and death. Concomitant resections of a C. septicum aortic aneurysm and colon cancer have also been successfully performed [3].

This case highlights the essential features of infectious aortitis caused by Clostridium septicum and the associated need for a high index of suspicion for rapid diagnosis and management. Prompt initiation of intravenous antibiotic therapy followed by consideration for early surgical intervention is essential to reduce the high mortality associated with this condition [5].

\section{Disclosure}

This case was presented in abstract format as part of the Society of Vascular Medicine meeting. Abstracts Submitted for the 2011 SVM 22nd Annual Meeting and Scientific Sessions: June 2-4, 2011 Boston, MA, USA, Vascular Medicine, 2011. This case was also presented at the 22nd society of vascular meeting Minneapolis, MN.

\section{References}

1. Smith-Slatas CL, Bourque M, Salazar JC. Clostridium septicum infections in children: a case report and review of the literature. Pediatrics. 2006;117(4):e796-805.

2. Koransky JR, Stargel MD, Dowell VR, Jr. Clostridium septicum bacteremia. Its clinical significance. Am J Med. 1979;66(1):63-66.

3. Seder CW, Kramer M, Long G, Uzieblo MR, Shanley CJ, Bove P. Clostridium septicum aortitis: Report of two cases and review of the literature. J Vasc Surg. 2009;49(5):1304-1309.

4. Moseley B, Mwirigi NW, Bowen J. Clostridium septicum Aortitis and Cecal Adenocarcinoma. Case Rep Med. 2010;2010:121728.

5. Sailors DM, Eidt JF, Gagne PJ, Barnes RW, Barone GW, McFarland DR. Primary Clostridium septicum aortitis: a rare cause of necrotizing suprarenal aortic infection. A case report and review of the literature. J Vasc Surg. 1996;23(4):714-718. 